\title{
Cryopreservation of Biologically Functional Submandibular Gland Rudiments from Fetal Mice
}

\author{
KEISUKE ADACHI ${ }^{1,2}$, YUTA OHNO ${ }^{2}$, KEITARO SATOH ${ }^{2}$, AKIKO SHITARA ${ }^{2}$, \\ YASUNORI MURAMATHU ${ }^{1}$ and MASANORI KASHIMATA ${ }^{2}$ \\ ${ }^{1}$ Department of Oral Maxillofacial Surgery, Asahi University School of Dentistry, Gifu, Japan; \\ ${ }^{2}$ Department of Pharmacology, Asahi University School of Dentistry, Gifu, Japan
}

\begin{abstract}
Background/Aim: Cryopreservation of cell lines has been widely used in the laboratory; however, cryopreservation of organs is still considered to be difficult. The submandibular gland (SMG) of fetal mice is one of the best-characterized organs. We investigated the conditions for cryopreserving SMG rudiments. Materials and Methods: Embryonic day 13 SMG rudiments were cryopreserved with or without a cryoprotectant. They were thawed and incubated in DMEM/F12 medium. Moreover, the influence of EGF stimulation on the signaling cascade after frozen-thawing the rudiments was analyzed by Western blotting. Results: When $S M G$ rudiments were cryopreserved without a cryoprotectant, all cells in the rudiments died. However, the SMG rudiments that had been preserved in a cryoprotectant showed branching morphogenesis. Additionally, the responsiveness of signaling cascades to EGF did not differ between frozen with a cryoprotectant and non-frozen rudiments. Conclusion: Cryopreservation might be a useful technology for preserving tissues from small organs, such as fetal SMG rudiments.
\end{abstract}

Cryopreservation of cell lines has been widely used in the laboratory for in vitro study of cell systems. Cryopreservation is also useful for germ cells in medical care, for organic long-term preservation of induced pluripotent stem cells (iPS cells), and in animal husbandry (1-4). When cells are frozen without using a cryoprotective agent (CPA), most of the constituent cells die. This is because the water molecules inside and outside of the cells

This article is freely accessible online.

Correspondence to: Masanori Kashimata, Department of Pharmacology, Asahi University School of Dentistry, 1851-1 Hozumi, Mizuho, Gifu 501-0296, Japan. Tel: +81 583291431, Fax: +81 583291432, e-mail: matasan@ dent.asahi-u.ac.jp

Key Words: Cryopreservation, branching morphogenesis, submandibular gland, EGF, ERK1/2, AKT. crystallize, and the cytoarchitecture, such as cell membranes, is physically destroyed $(5,6)$. CPAs contain chemical compounds, such as dimethyl sulfoxide (DMSO), glycerol, sucrose and ethylene glycol, and the compositions of CPAs differ depending on the cells to be frozen (5). Despite all the available protocols for successful cryopreservation of different cell types, cryopreservation of organs and tissues is still considered difficult.

Mouse SMG rudiment development begins on embryonic day 12 (E12) when the epithelial tissue of the floor of the mouth sinks towards the direction of the neck. On E13, the SMG rudiment is oval shaped, approximately $500 \mu \mathrm{m}$, and it rapidly forms a glandular body by repeated cleft formation and stalk elongation, called branching morphogenesis (BrM). $\mathrm{BrM}$ is studied in SMG rudiments on filters floating in serum-free medium in ex vivo organ culture systems (7-10). The epithelial cells constituting SMG rudiments promote BrM when stimulated with cell growth factors. Kashimata, et al. (1997) have reported that epidermal growth factor (EGF) strongly promotes BrM of the SMG epithelium (11), while Hoffman et al. (2002) have reported that fibroblast growth factor (FGF) also promotes BrM of the SMG epithelium (12). EGF and FGF are secreted mainly from the mesenchyme, and growth factor receptors are expressed in the epithelial cells of SMG rudiments $(12,13)$. Following binding of EGF or FGF to their respective receptors, signaling pathways, such as extracellular signal-regulated kinase-1/2 (ERK1/2), classical mitogen-activated protein kinase (MAPK) and phosphoinositide 3-kinase (PI3K)-AKT cascades are activated (11-15). Therefore, EGF and FGF induce cell proliferation and differentiation in the developing mouse SMG through activation of these signaling cascades (11-15).

Recently, Ogawa et al. (2013) prepared SMGs from immature epithelial and mesenchymal cells obtained from fetal mouse SMGs and transplanted them into mice whose salivary glands had been removed (16). They, then, demonstrated secretion of saliva from the transplanted SMGs. Since steady progress is being made in regenerative therapy of organs, including the salivary gland, the need for 
A

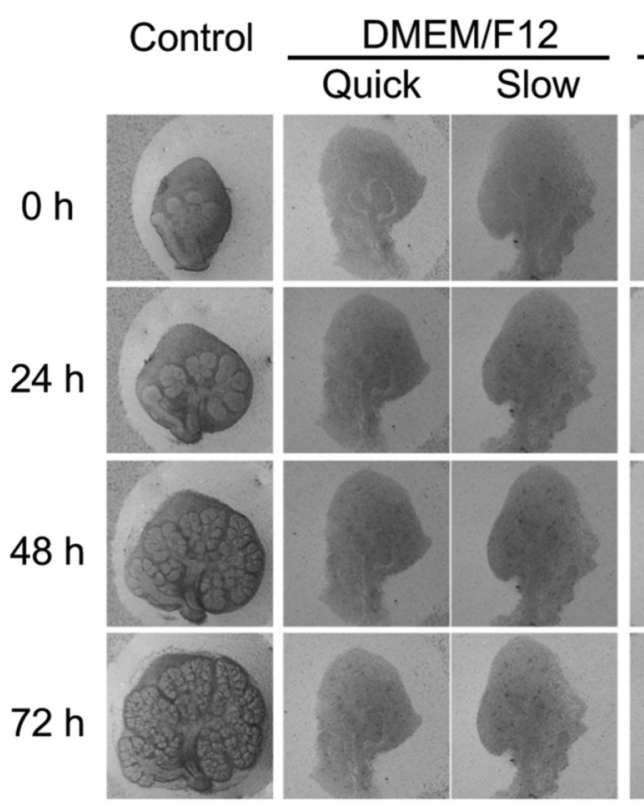

C $10 \%$ DMSO
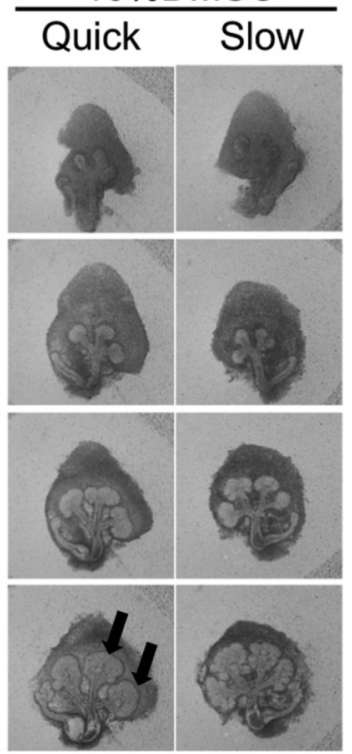

D
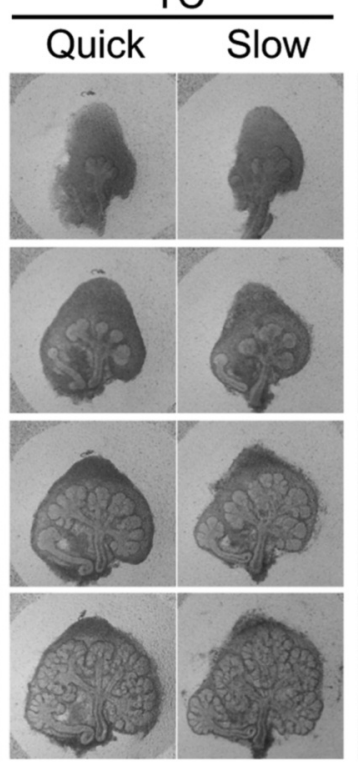

$\mathbf{E}$
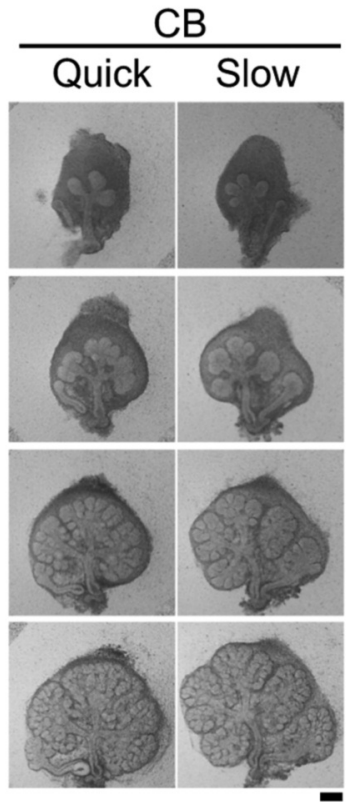

Figure 1. Morphological changes in cultured SMG rudiments. (A) E13 non-frozen control SMG rudiments floating in DMEM/F12 medium. (B-E) $S M G$ rudiments cryopreserved at $-80^{\circ} \mathrm{C}$ for 24 h either in a freezing tube box (Quick) or a Mr. Frosty (Slow) with (B) DMEM/F12, (C) DMEM/F12 medium containing 10\% DMSO, (D) TC only, and (E) CB only. All SMG rudiments were cultured for 0-72 h. Some of cryoprotective agents (CPA) were protected the cells of $S M G$ rudiments although the protective effects were different from each other. The black arrow indicates a hypertrophy-

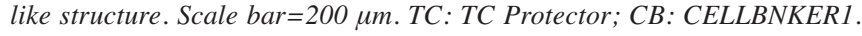

further development of cryopreservation technologies for organs will almost certainly increase in the future.

In this study, we investigated whether CPAs protect cryopreserved SMG rudiments and induce the morphological changes of BrM in thawed and incubated rudiments, and whether EGF-responsive phosphorylation of ERK1/2 and AKT occurs in SMG rudiments cryopreserved with CPAs.

\section{Materials and Methods}

Cryopreservation of SMG rudiments. Pregnant mice (ICR strain) were purchased from Japan SLC (Hamamatsu, Japan). The day of discovery of a vaginal plug was taken as embryonic day 0 (E0). E13 SMG rudiments were removed from fetuses as described previously $(13,17)$. The SMG rudiments were placed in 4 different CPAs: i) DMEM/F12 medium (Gibco Invitrogen Cell Culture, Carlsbad, CA, USA) containing 100 units $/ \mathrm{ml}$ penicillin (Gibco), $100 \mu \mathrm{g} / \mathrm{ml}$ streptomycin (Gibco), $150 \mu \mathrm{g} / \mathrm{ml}$ vitamin C (Sigma-Aldrich, St. Louis, MO, USA), and $50 \mu \mathrm{g} / \mathrm{ml}$ transferrin (Sigma-Aldrich), ii) DMEM/F12 containing 10\% DMSO (Sigma-Aldrich), iii) TC Protector (TC) (DS pharma Biomedical Co., Osaka, Japan), and iv) CELLBANKER1 (CB) (ZENOAQ, Fukushima, Japan). One SMG rudiment was put into $200 \mu \mathrm{l}$ of each CPA solution in a cryogenic tube (Thermo Fisher Scientific, Waltham, MA, USA) and placed in frozen tube boxes (quick freezing) or in a Mr. Frosty (slow freezing,
Thermo Fisher Scientific) and then stored in a deep freezer at $-80^{\circ} \mathrm{C}$ for $24 \mathrm{~h}$. Mr. Frosty is a container whose inner temperature slowly decreases at a rate of $1^{\circ} \mathrm{C} / \mathrm{min}$ in a $-80^{\circ} \mathrm{C}$ deep freezer. All animal handling procedures were conducted in accordance with the Guideline for Experimental Animals of the Asahi University (Protocol No. 18-007).

Culture of cryopreserved SMG rudiments. The frozen SMG rudiments were quickly thawed by adding $900 \mu \mathrm{l}$ of $37^{\circ} \mathrm{C}$ DMEM/F12 medium, followed by pipetting. Then the SMG rudiments were washed two times with $1 \mathrm{ml}$ of DMEM/F12 medium, placed on a membrane filter (Nuclepore membrane, 0.1 $\mu \mathrm{m}$ pore size; Whatman International, Brantford, UK) floating in $400 \mu \mathrm{l}$ of DMEM/F12 medium, and cultured in $5 \% \mathrm{CO}_{2}, 95 \%$ air and $80 \%$ humidity at $37^{\circ} \mathrm{C}$ ex vivo. The cultured SMG rudiments were photographed at $0,24,48$ and $72 \mathrm{~h}$ following incubation. Subsequently, the endpieces of the SMG rudiments were counted, and the area of the epithelium of the SMG rudiments was measured. As a non-frozen control, normal E13 SMG rudiments were held at $4^{\circ} \mathrm{C}$ for $24 \mathrm{~h}$, cultured and photographed. The number of endpieces and the area of the epithelium of these non-frozen control SMG rudiments were used as control values.

Western blotting for signaling proteins. E13 SMG rudiments were cryopreserved in $\mathrm{CB}$ and thawed $24 \mathrm{~h}$ later, as described above. The cryopreserved SMG rudiments and non-frozen control SMG 
A

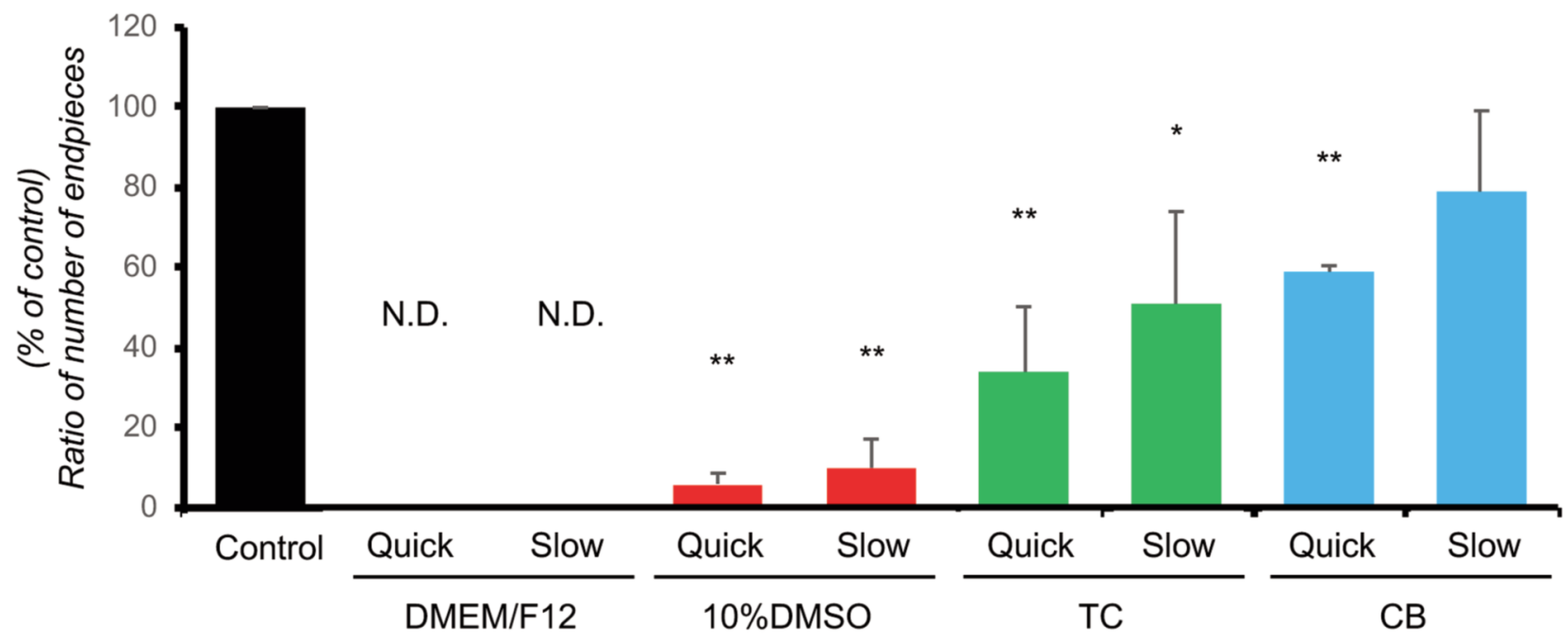

B

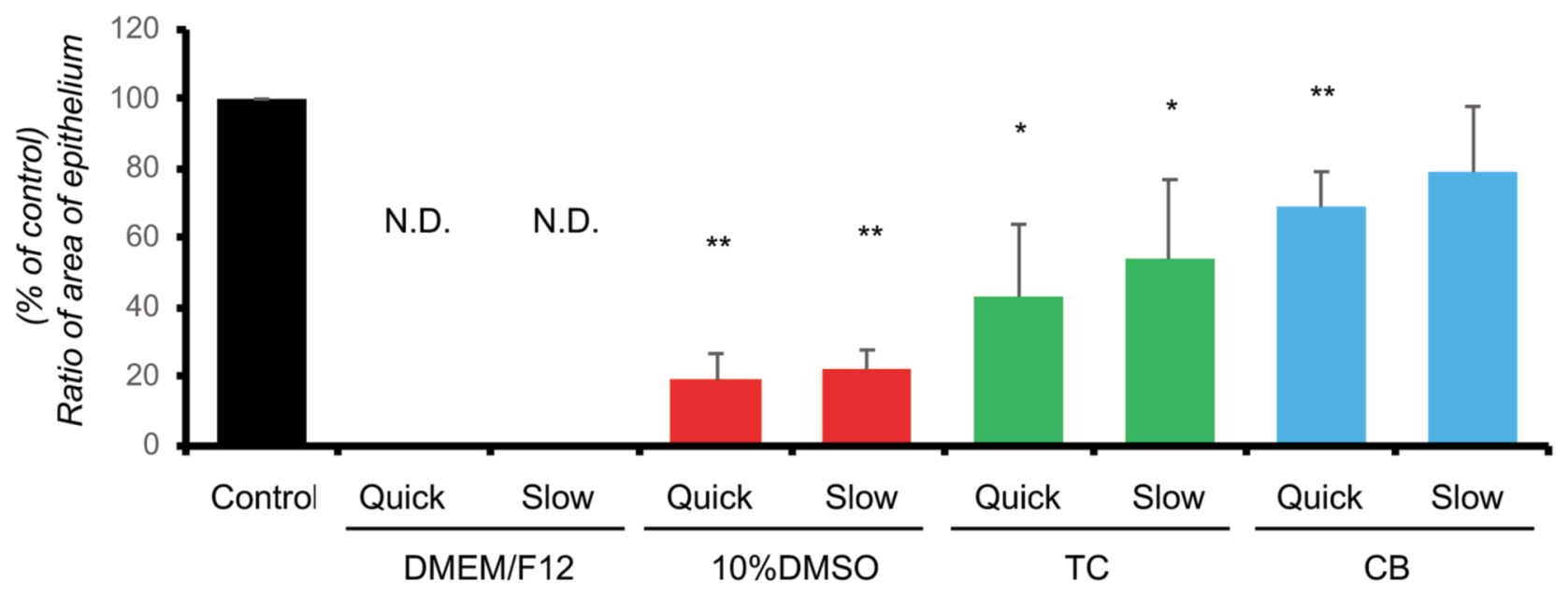

Figure 2. Influence of cryoprotective agents on branching morphogenesis of cultured SMG rudiments. The number of endpieces (A) and the area of the epithelium (B) of E13 cultured SMG rudiments held under the different conditions were determined. All SMG rudiments were cultured for $72 \mathrm{~h}$. Values represent the mean $\pm S D$ of four independent experiments. N.D.: not detected; Quick: quick freezing (using a freezing tube box); Slow: slow freezing (using a Mr. Frosty). *p<0.05, **p<0.01.

rudiments were cultured on membrane filter for $24 \mathrm{~h}$, as described above. Subsequently, EGF $20 \mathrm{ng} / \mathrm{ml}$ (R \& D System Inc., Minneapolis, MN, USA) was added to the medium, followed by incubation for 0,10 or $30 \mathrm{~min}$. The rudiments were washed with ice-cold PBS containing $1 \mathrm{mM} \mathrm{Na} \mathrm{VO}_{4}$ and phenylmethylsulfonyl fluoride (PMSF, Sigma-Aldrich), and homogenized with cell lysis buffer (Cell Signaling Technology Inc.). Centrifugation was performed at $15,000 \mathrm{rpm}$ for $30 \mathrm{~min}$ at $4^{\circ} \mathrm{C}$, and the resulting supernatants were collected. The protein concentrations were measured using the Pierce $660 \mathrm{~nm}$ Protein Assay Reagent (Thermo Fisher Scientific). Aliquot protein samples $(2 \mu \mathrm{g})$ were subjected to SDS-PAGE and transferred to polyvinylidene difluoride (PVDF) membranes (BIO-RAD, Hercules, CF, USA). Specific proteins on the membrane were detected by probing with the specific primary monoclonal or polyclonal antibodies described above, followed by secondary antibodies conjugated to horseradish peroxidase. The proteins were detected using ECL Select Western Blotting Detection Reagents (Amersham Biosciences, Buckinghamshire, UK). The intensities of the Western blot bands were analyzed using a CS analyzer (ATTO Corp., Tokyo, Japan). All Western blots were repeated at least three times.

Statistical analysis. One-way ANOVA followed by Dunnettt's multiple comparison test was used for comparing experimental groups with the control. Values of $p<0.05$ and $p<0.01$ were considered to be statistically significant. 


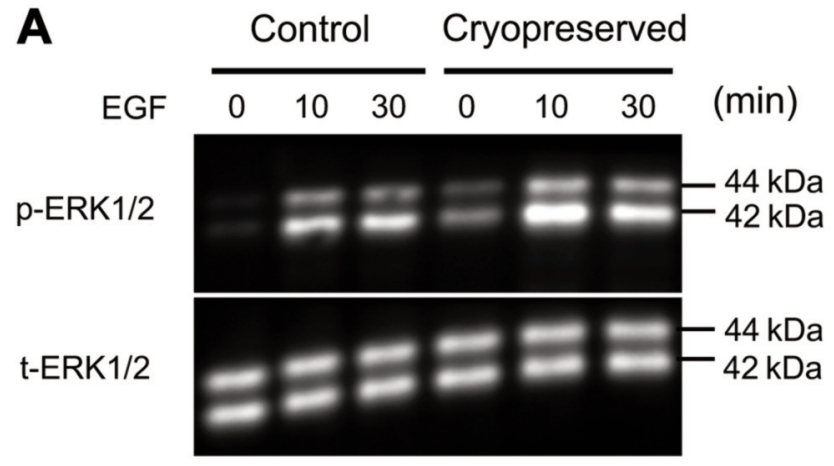

B

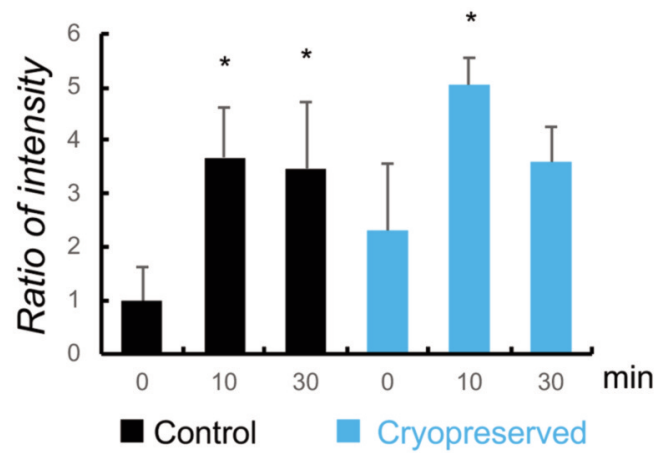

Figure 3. Western blot analysis of ERK1/2 phosphorylation by EGF in cultured SMG rudiments with or without cryopreservation. E13 SMG rudiments were collected and separated into two groups. The control group was not frozen but was held at $4^{\circ} \mathrm{C}$ for $24 \mathrm{~h}$, and the other group was cryopreserved in $\mathrm{Mr}$. Frosty at $-80^{\circ} \mathrm{C}$ for $24 \mathrm{~h}$ in $\mathrm{CB}$ as a CPA. $S M G$ rudiments were cultured and stimulated with EGF $(20 \mathrm{ng} / \mathrm{ml})$ for 0,10 , and $30 \mathrm{~min}$. The SMG rudiments were homogenized, and the resulting supernatants were analyzed by Western blotting. (A) Upper panel: Phosphorylated ERK1/2 (p-ERK1/2); lower panel: Total ERK1/2 $(t$-ERK1/2). (B) The intensities of the Western blot bands were analyzed using a CS analyzer and expressed as a ratio ( $p E R K 1 / 2$ per $t$-ERK1/2 at each incubation time). Values represent the mean $\pm S D$ of three independent experiments. ANOVA compared with 0 min. ${ }^{*} p<0.05$.

\section{Results}

Culture of cryopreserved SMG rudiments. Figure 1 shows the morphological changes in cultured rudiments of non-frozen control SMGs and thawed SMGs that had been cryopreserved at $-80^{\circ} \mathrm{C}$ for $24 \mathrm{~h}$ in each of the 4 CPAs. The area of nonfrozen control E13 SMG rudiments increased (Figure 1A), whereas SMG rudiments that had been cryopreserved in $\mathrm{DMEM} / \mathrm{F} 12$ medium at $-80^{\circ} \mathrm{C}$ for $24 \mathrm{~h}$ showed no changes at all under both quick- and slow-freezing conditions (Figure 1B). The number of endpieces and the area of the epithelium in cultured rudiments that had been cryopreserved in DMEM/F12 medium at $-80^{\circ} \mathrm{C}$ for $24 \mathrm{~h}$ could not be determined under both quick- and slow-freezing conditions, as the border between the epithelium and mesenchyme of SMG rudiments cryopreserved without any CPA disappeared (Figures 2A and B). However,

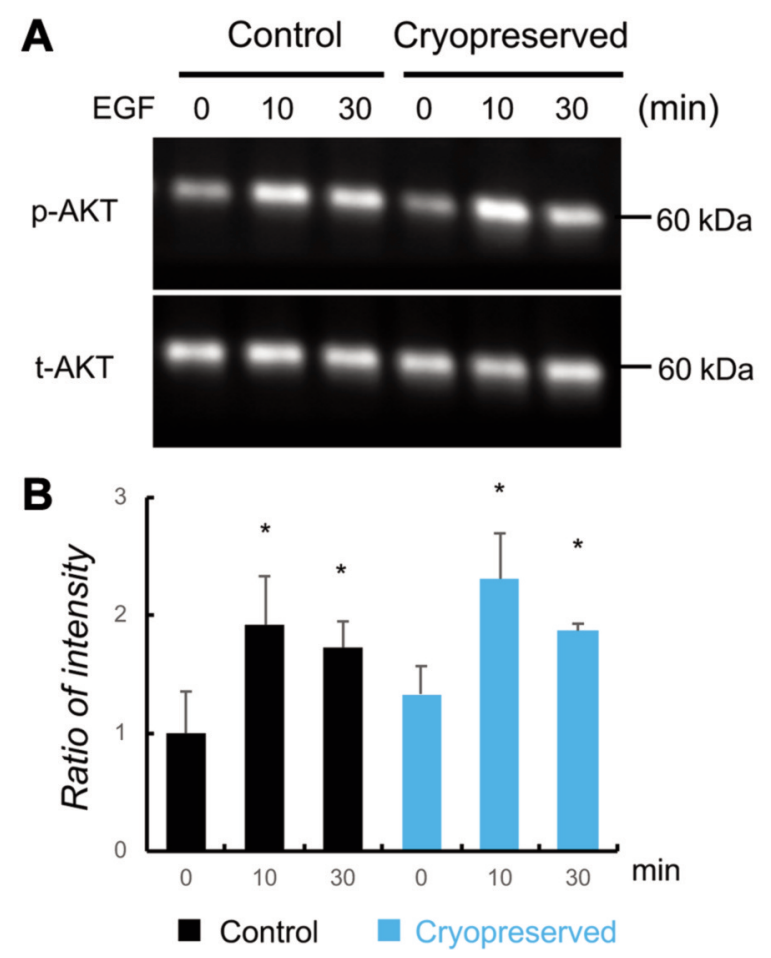

Figure 4. Western blot analysis of AKT phosphorylation by EGF in cultured SMG rudiments with or without cryopreservation. E13 SMG rudiments were collected and separated into two groups. The control group was held at $4^{\circ} \mathrm{C}$ for $24 \mathrm{~h}$, and the other group was cryopreserved in a Mr. Frosty at $-80^{\circ} \mathrm{C}$ for $24 \mathrm{~h}$ in $C B$ as a CPA. The SMG rudiments were cultured and stimulated with EGF (20 $\mathrm{ng} / \mathrm{ml})$ for 0, 10 and $30 \mathrm{~min}$. The SMG rudiments were homogenized, and the resulting supernatants were analyzed by Western blotting. (A) Upper panel: Phosphorylated $A K T$ ( $p$-AKT); lower panel: Total AKT (t-AKT). (B) The intensities of the Western blot bands were analyzed using a CS analyzer and expressed as a ratio ( $p$-AKT per $t$-AKT at each incubation time). Values reprsent the mean $\pm S D$ of three independent experiments. ANOVA compared with $0 \mathrm{~min},{ }^{*} p<0.05$.

the SMG rudiments cryopreserved in $10 \%$ DMSO under both quick- and slow-freezing conditions grew slowly during incubation (Figure 1C) compared to the non-frozen control SMG rudiments (Figure 1A). Therefore, some epithelial cells and mesenchymal cells in the rudiments cryopreserved in $10 \%$ DMSO had remained alive, and BrM occurred during incubation (Figure 1C), even though BrM appeared abnormal, with smaller and fewer epithelial endpieces in the rudiments, resulting in hypertrophy. The number of endpieces and the area of the epithelium of the rudiments cryopreserved in $10 \%$ DMSO increased under both quick- and slow-freezing conditions (Figures 2A and B), and those values differed significantly compared to the non-frozen control SMG rudiments $(p<0.01)$.

The protective effects of TC and $\mathrm{CB}$ as CPAs for SMG rudiments were stronger than that of $10 \%$ DMSO. As shown 
in Figure 1, the morphological changes of SMG rudiments cryopreserved in TC and CB under both quick- and slowfreezing conditions were similar to the changes in the nonfrozen control SMG rudiments during 72-h culture (Figure 1A). The number of endpieces and the epithelial area of cultured SMG rudiments after cryopreservation in TC and CB (Figure 2A) under both quick- and slow-freezing conditions were smaller compared to the non-frozen control SMG rudiments. There were significant differences in the number of endpieces between SMGs cryopreserved in TC under quick-frozen and non-frozen control SMGs $(p<0.01)$, between SMGs cryopreserved in TC under slow-frozen and non-frozen control SMGs $(p<0.05)$, and between SMGs cryopreserved in $\mathrm{CB}$ under quick-frozen and non-frozen control SMGs $(p<0.01)$. However, no significant differences were seen between the number of endpieces in SMGs cryopreserved in $\mathrm{CB}$ under slow-freezing and non-freezing conditions and in the control SMGs (Figure 2A). Also, no significant differences were seen between the epithelial area of the SMGs cryopreserved in $\mathrm{CB}$ under slow-freezing conditions and the non-frozen control SMGs (Figure 2B). On the other hand, there were significant differences between SMGs cryopreserved in TC under quick- and slow-freezing conditions and the non-frozen control SMGs $(p<0.05)$, and between SMGs cryopreserved in CB under quick-freezing conditions and the non-frozen control SMGs $(p<0.01)$. This means that BrM of SMG rudiments cryopreserved at $-80^{\circ} \mathrm{C}$ for $24 \mathrm{~h}$ in $\mathrm{CB}$ under slow-freezing conditions was normal, similar to the non-frozen control SMGs. In particular, following a 24-h culture cryopreservation in CB, the SMG rudiments looked healthier compared to the other cryopreserved rudiments (Figure 1E).

Cell signaling systems of cryopreserved SMG rudiments. It is well known that EGF stimulates ERK1/2 and AKT phosphorylation and induces BrM in cultured SMG rudiments $(15,17,18)$. We investigated whether functional ERK1/2 and AKT signaling cascades remained activated in cryopreserved SMG rudiments. As shown in Figure 3A, ERK1/2 in cultured non-frozen control SMG rudiments was phosphorylated at 10 and 30 min following the addition of EGF. EGF increased the phosphorylation levels significantly, by more than 3 -fold $(p<0.01$, Figure $3 \mathrm{~B})$. EGF also stimulated ERK1/2 phosphorylation in the cultured SMG rudiments after cryopreservation in $\mathrm{CB}$ (Figure $3 \mathrm{~A}$ ). The levels of phosphorylation increased by 2.2 -fold at $10 \mathrm{~min}$ and by 1.6 -fold at $30 \mathrm{~min}$, respectively ( $p<0.05$, Figure $3 \mathrm{~B}$ ).

Figure 4 shows our findings regarding the AKT cascade in the SMG rudiments. EGF stimulated AKT phosphorylation in the cultured non-frozen control SMG rudiments. The levels of phosphorylation were increased 1.9fold at $10 \mathrm{~min}$ and 1.7-fold at $30 \mathrm{~min}$ after EGF stimulation $(p<0.05$, Figure 4B). EGF stimulation also increased AKT phosphorylation in CB-cryopreserved SMG rudiments, by 1.7 -fold at $10 \mathrm{~min}$ and 1.4 -fold at $30 \mathrm{~min}(p<0.05$, Figure $4 \mathrm{~B})$. These results show that functional, EGF-responsive ERK1/2 and AKT signaling cascades remain active in SMG rudiments cryopreserved in $\mathrm{CB}$.

\section{Discussion}

Cryopreservation methods have already been developed for mammalian cell lines and are widely used in the laboratory for decades. Cryopreservation is also used for oocytes, embryonic stem (ES) cells and iPS cells (1-3). CPAs, such as DMSO, glycerol, sucrose and polyethylene glycol are available for cryopreservation of many types of cells (5). However, there is no general method of cryopreservation for tissues, except for testicular and ovarian tissues $(19,20)$. Therefore, cryopreservation of organs and/or organ rudiments remains difficult.

The fetal mouse SMG is a useful model for studying organogenesis, epithelial-mesenchymal interactions and $\mathrm{BrM}$, and it is able to undergo BrM even in ex vivo systems (21-23). Since BrM, which is driven by epithelialmesenchymal interactions, can be observed in the culture systems of SMG rudiments, there is no need for addition of cell growth factors or serum $(18,24,25)$. We tried to determine the damage caused by cryopreservation of organs and the effects of various CPAs on epithelial BrM using incubated SMG rudiments.

Most of the cells in SMG rudiments that were frozen without any CPA died. The border between the epithelium and mesenchyme of SMG rudiments cryopreserved without any CPA was found to have disappeared when they were cultured ex vivo. However, when cryopreserved in $10 \%$ DMSO, some cells in the SMG rudiments survived, and a few epithelial endpieces of cryopreserved SMG rudiments were observed after $24 \mathrm{~h}$ in culture; and even increased during longer culture periods. This means that not only the cells of the epithelium and mesenchyme of the SMG rudiments, but also the 3D structure of the extracellular matrix, was partly protected by $10 \%$ DMSO. DMSO has been previously reported to be effective for the cryopreservation of cell lines $(5,26)$. Our present results show that DMSO is also effective for the cryopreservation of SMG rudiments. However, the morphological changes were quite different compared to the non-frozen control SMG rudiments. The rate of BrM was very slow in culture until $24 \mathrm{~h}$, and a hypertrophylike structure was observed in endpieces of the SMG epithelium, probably due to inhibition of cleft formation and decreased numbers of endpieces.

The protective effects of TC and CB as CPAs were greater than that of the $10 \%$ DMSO. The morphological changes in cultured SMG rudiments that had been 
cryopreserved in TC and $\mathrm{CB}$ were very similar to those of the non-frozen control rudiments. However, the morphological changes after $72 \mathrm{~h}$ of culture were totally different compared to those of the 10\% DMSO group, although the SMG rudiments cryopreserved in TC showed a slower rate of BrM. The results for the area of the epithelium and the number of endpieces of the cultured SMG rudiments did not differ significantly between the non-frozen control SMG rudiments and the SMG rudiments cryopreserved in $\mathrm{CB}$ by slow freezing in a Mr. Frosty. Although TC and CB are sold worldwide as CPAs for the cryopreservation of cell lines, their ingredients are not disclosed. The reagent descriptions state that $\mathrm{CB}$ contains serum but TC does not. The difference in protective effect between $\mathrm{CB}$ and TC may, thus, be due to the presence or absence of serum.

BrM of SMG rudiments is regulated by many factors, including cell growth factors, adhesion molecules and extracellular matrix $(23,27)$. These factors are known to stimulate intracellular signaling cascades, including mitogenactivated protein kinase (MAPK) and AKT. We investigated whether these cell signaling cascades were damaged by the cryopreservation of SMG rudiments. Western blotting analysis clearly showed that EGF stimulated the phosphorylation of ERK1/2 in SMG rudiments cryopreserved in $\mathrm{CB}$ in a Mr. Frosty. A similar result was obtained for AKT phosphorylation. Although the phosphorylation levels of ERK1/2 and AKT by EGF in cryopreserved rudiments were somewhat lower compared to the levels of the non-frozen control SMG rudiments, the EGF-responsive activation systems for both signaling cascades were at least still present, even after cryopreservation. There have been few research reports on cryopreservation of organs to date. In this study, slow freezing in CB significantly protected the biological functions of SMG rudiments from fetal mice.

Our present findings open the possibility that larger or mature organs may be effectively cryopreserved subsequent to further development of suitable cryopreservation reagents and freezing conditions.

\section{Conflicts of Interest}

The Authors have no conflicts of interest to declare regarding this study.

\section{Authors' Contributions}

KA and MK prepared cryopreservation of SMG rudiments. KA, YM and MK performed the experiments of culture of frozen SMG rudiments. YO and KS performed the western blotting and analyzed the data. AS Performed morphological analyses of cultured SMG rudiments. All authors (KA, YO, KS, AS, YM and MK) organized the research and wrote the manuscript.

\section{Acknowledgements}

This work was supported by JSPS KAKENHI Grant Number 19K10193 and a Miyata Grant for Scientific Research (B) from Asahi University.

\section{References}

1 Kuwayama M: Highly efficient vitrification for cryopreservation of human oocytes and embryos: the Cryotop method. Theriogenology 67(1): 73-80, 2007. PMID: 17055564. DOI: 10.1016/j.theriogenology.2006.09.014

2 Hunt CJ: Cryopreservation of human stem cells for clinical application: A review. Transfus Med Hemother 38(2): 107-123, 2011. PMID: 21566712. DOI: $10.1159 / 000326623$

3 Mandawala AA, Harvey SC, Roy TK and Fowler KE: Cryopreservation of animal oocytes and embryos: Current progress and future prospects. Theriogenology 86(7): 1637-1644, 2016. PMID: 27555525. DOI: 10.1016/j.theriogenology. 2016.07.018

4 Morrell JM and Mayer I: Reproduction biotechnologies in germplasm banking of livestock species: a review. Zygote 25(5): 545-557, 2017. PMID: 28835292. DOI: 10.1017/ S0967199417000442

5 Rapats G and Luyet B: Patterns of ice formation in aqueous solutions of glycerol. Biodynamica 10(198): 1-68, 1966. PMID: 5971632.

6 Mazur P: Cryobiology: the freezing of biological systems. Science 168(3934): 939-949, 1970. PMID: 5462399.

7 Borghese E: The development in vitro of the submandibular and sublingual glands of Mus musculus. J Anat 84(3): 287-302, 1950. PMID: 15436333

8 Borghese E: Explantation experiments on the influence of the connective tissue capsule on the development of the epithelial part of the submandibular gland of Mus musculus. J Anat 84(3): 303-318, 1950. PMID: 15436334.

9 Spooner BS, Thompson-Pletscher HA, Stokes B and Bassett KE: Extracellular matrix involvement in epithelial branching morphogenesis. Dev Biol (N Y 1985) 3: 225-260, 1986. PMID: 3077966. DOI: 10.1007/978-1-4684-5050-7_12

10 Cutler LS and Gremski W: Epithelial-mesenchymal interactions in the development of salivary glands. Crit Rev Oral Biol Med 2(1): 1-12, 1991. PMID: 1912140. DOI: 10.1177/ 10454411910020010101

11 Kashimata M and Gresik EW: Epidermal growth factor system is a physiological regulator of development of the mouse fetal submandibular gland and regulates expression of the alpha6integrin subunit. Dev Dyn 208(2): 149-161, 1997. PMID: 9022052. DOI: 10.1002/(SICI)1097-0177(199702)208:2<149:: AID-AJA2>3.0.CO;2-I

12 Hoffman MP, Kidder BL, Steinberg ZL, Lakhani S, Ho S, Kleinman HK and Larsen M: Gene expression profiles of mouse submandibular gland development: FGFR1 regulates branching morphogenesis in vitro through BMP- and FGF-dependent mechanisms. Development 129(24): 5767-5778, 2002. PMID: 12421715. DOI: $10.1242 / \mathrm{dev} .00172$

13 Mizukoshi K, Koyama N, Hayashi T, Zheng L, Matsuura S and Kashimata M: Shh/Ptch and EGF/ErbB cooperatively regulate branching morphogenesis of fetal mouse submandibular glands. 
Dev Biol 412(2): 278-287, 2016. PMID: 26930157. DOI: 10.1016/j.ydbio.2016.02.018

14 Gresik EW, Kashimata M, Kadoya Y, Mathews R, Minami N and Yamashina S: Expression of epidermal growth factor receptor in fetal mouse submandibular gland detected by a biotinyltyramide-based catalyzed signal amplification method. J Histochem Cytochem 45(12): 1651-1657, 1997. PMID: 9389768. DOI: $10.1177 / 002215549704501208$

15 Kashimata M, Sayeed S, Ka A, Onetti-Muda A, Sakagami H, Faraggiana $\mathrm{T}$ and Gresik EW: The ERK-1/2 signaling pathway is involved in the stimulation of branching morphogenesis of fetal mouse submandibular glands by EGF. Dev Biol 220(2): 183-196, 2000. PMID: 10753509. DOI: 10.1006/dbio.2000.9639

16 Ogawa M, Oshima M, Imamura A, Sekine Y, Ishida K, Yamashita K, Nakajima K, Hirayama M, Tachikawa T and Tsuji T: Functional salivary gland regeneration by transplantation of a bioengineered organ germ. Nat Commun 4: 2498, 2013. PMID: 24084982. DOI: 10.1038/ncomms3498

17 Koyama N, Hayashi T, Ohno K, Siu L, Gresik EW and Kashimata M: Signaling pathways activated by epidermal growth factor receptor or fibroblast growth factor receptor differentially regulate branching morphogenesis in fetal mouse submandibular glands. Dev Growth Differ 50(7): 565-576, 2008. PMID: 19238727. DOI: 10.1111/j.1440-169x.2008.01053.x

18 Larsen M, Hoffman MP, Sakai T, Neibaur JC, Mitchell JM and Yamada KM: Role of PI 3-kinase and PIP3 in submandibular gland branching morphogenesis. Dev Biol 255(1): 178-191, 2003. PMID: 12618142. DOI: 10.1016/s0012-1606(02)00047-7

19 Hovatta O: Cryopreservation of testicular tissue. Mol Cell Endocrinol 169(1-2): 113-115, 2000. PMID: 11155942. DOI: 10.1016/s0303-7207(00)00363-4

20 Rivas Leonel EC, Lucci CM and Amorim CA: Cryopreservation of human ovarian tissue: A review. Transfus Med Hemother 46(3): 173-181, 2019. PMID: 31244585. DOI: 10.1159/ 000499054
21 Takahashi Y and Nogawa H: Branching morphogenesis of mouse salivary epithelium in basement membrane-like substratum separated from mesenchyme by the membrane filter. Development 111(2): 327-335, 1991. PMID: 1893867.

22 Tucker AS: Salivary gland development. Semin Cell Dev Biol 18(2): 237-244, 2007. PMID: 17336109. DOI: 10.1016/j. semcdb.2007.01.006

$23 \mathrm{Hsu}$ JC and Yamada KM: Salivary gland branching morphogenesis-recent progress and future opportunities. Int J Oral Sci 2(3): 117-126, 2010. PMID: 21125789. DOI: 10.4248/ IJOS 10042

24 Nogawa H and Takahashi Y: Substitution for mesenchyme by basement-membrane-like substratum and epidermal growth factor in inducing branching morphogenesis of mouse salivary epithelium. Development 112(3): 855-861, 1991. PMID: 1935691.

25 Sakai T: Epithelial branching morphogenesis of salivary gland: exploration of new functional regulators. $J$ Med Invest 56(Suppl): 234-238, 2009. DOI: 10.2152/jmi.56.234

26 Ashwood-Smith MJ: Genetic damage is not produced by nomal cryopreservation procedures involving either glycerol or dimethyl sulfoxide: a cautionary note, however, on possible effects of dimethyl sulfoxide. Cryobiology 22(5): 427-433, 1985. PMID: 3902368. DOI: 10.1016/0011-2240(85)90153-1

27 Kashimata $M$ and Hayashi T: Regulatory mechanisms of branching morphogenesis in mouse submandibular gland rudiments. Jpn Dent Sci Rev 54(1): 2-7, 2018. PMID: 29628996. DOI: $10.1016 /$ j.jdsr.2017.06.002
Received July 29, 2020

Revised August 10, 2020

Accepted August 11, 2020 